\title{
Production et composition du lait de brebis : effets de l'apport de lipides protégés
}

\author{
A. Gargouri ${ }^{1}$
}

Mots-clés

Ovin - Lait de brebis - Lipide Composition chimique - Agneau Croissance - Tunisie.

\begin{abstract}
Résumé
Une base de données sur les effets de l'apport des lipides protégés (savons de calcium à base d'huile de palme principalement) a été constituée pour qualifier la réponse de la production et de la composition du lait chez les brebis laitières. D'une façon générale, l'incorporation de ce type de supplément n'affecte pas la quantité de lait commercialisable, entraîne une augmentation du taux butyreux (+ 21 p. 100) et une dépression du taux protéique ( -4 p. 100). Ces réponses sont très variables et dépendent de la quantité de supplément lipidique et du stade de lactation des brebis. Les meilleures réponses sont observées avec un niveau d'ingestion de savon calcique proche de 150 g/jour/ brebis administrés en début de lactation. La composition en acides gras du lait est presque toujours modifiée par les lipides protégés, avec notamment une diminution de la proportion des acides gras courts et moyens, et un accroissement des acides gras longs. La vitesse de croissance des agneaux élevés par les brebis supplémentées n'est pas modifiée durant la période d'allaitement.
\end{abstract}

\section{INTRODUCTION}

L'utilisation des lipides protégés dans la ration des brebis, par analogie avec ce qui se fait chez les vaches laitières, devient de plus en plus fréquente en élevage ovin. Les objectifs, au-delà d'augmenter la densité énergétique de la ration, en particulier en début de lactation, sont d'accroitre la quantité totale de matière utile sécrétée dans le lait et par conséquent le rendement fromager, et d'agir sur les propriétés technologiques et organoleptiques des produits par la modification de la composition en acides gras du lait par transfert direct de certains acides gras issus des matières grasses protégées $(4,24,29)$.

Cependant, les études concernant l'incorporation des lipides protégés dans la ration des brebis sont beaucoup moins nombreuses que celles qui existent pour les vaches laitières. Néanmoins, quelques équipes, surtout espagnoles, se sont intéressées à ces questions. L'objectif de ce travail de synthèse a été de dégager les lois

1. Faculté des Sciences, département des Sciences de la vie, BP 802, 3018 Sfax, Tunisie

Tél. : +21698298153; fax : +21674274437

E-mail : Ahmed.Gargouri@fss.rnu.tn de réponse de l'apport des lipides protégés, essentiellement savons de calcium d'acides gras (Scag), dans la ration des brebis laitières à partir d'une base de données bibliographiques.

\section{MATERIEL ET METHODES}

\section{Réalisation de la base bibliographique}

La base bibliographique a été constituée en prenant les publications écrites par 11 auteurs différents, de 1986 à 2004. Ainsi, ont été retenus dans cette base tous les articles publiés comparant un traitement « témoin non complémenté par les lipides protégés » à au moins un traitement « complémenté » chez les brebis en production. Au total, 18 essais ont répondu à ce critère et ont été intégrés à la base qui totalise ainsi 46 réponses de production laitière, 47 réponses de taux butyreux et 43 réponses de taux protéique. Les principaux résultats zootechniques émanant de ces études sont résumés dans le tableau I. La réponse d'un «paramètre » à l'apport de lipides protégés a été définie comme la différence de ce paramètre entre le lot expérimental et le lot témoin non complémenté. Cette variable, nommée « delta paramètre » et notée $\Delta \mathrm{P}(\Delta$ Lait pour la production laitière brute, $\Delta \mathrm{TB}$ pour le taux butyreux et $\Delta \mathrm{TP}$ pour le taux protéique), a été la principale variable analysée. 


\section{Tableau I}

Effet de l'apport de savons de calcium d'acides gras (Scag) sur la production et la composition du lait de brebis pendant les phases d'allaitement et traite

\begin{tabular}{|c|c|c|c|c|c|c|c|c|c|c|c|c|}
\hline \multirow[t]{2}{*}{ Référence } & \multirow[t]{2}{*}{ Race } & \multirow[t]{2}{*}{ Etat } & \multirow{2}{*}{$\begin{array}{l}S L \\
\text { (j) }\end{array}$} & \multirow{2}{*}{$\begin{array}{c}\text { Conc. } \\
\text { (g/j) }\end{array}$} & \multicolumn{2}{|c|}{ Scag } & \multicolumn{2}{|c|}{ Production } & \multicolumn{4}{|c|}{ Composition } \\
\hline & & & & & $(g / j)$ & $\% \mathrm{CC}$ & Lait (ml/j) & $\Delta$ Lait & TB (g/l) & $\Delta \mathrm{TB}$ & $\mathrm{TP}(\mathrm{g} / \mathrm{l})$ & $\Delta \mathrm{TP}$ \\
\hline \multirow{4}{*}{$\begin{array}{l}\text { Appedu } \\
\text { et coll., } \\
2004\end{array}$} & \multirow[t]{4}{*}{ PP 1} & \multirow[t]{4}{*}{ Allaitement } & \multirow[t]{4}{*}{28} & \multirow[t]{2}{*}{ - } & 0 & 0 & 2480 & - & 99,0 & - & 47,0 & - \\
\hline & & & & & 100 & - & 2390 & -90 & 121,0 & 22,0 & 46,0 & $-1,0$ \\
\hline & & & & \multirow[t]{2}{*}{-} & 0 & 0 & 2230 & - & 83,0 & - & 47,0 & - \\
\hline & & & & & 116 & - & 2330 & 100 & 114,0 & 31 & 46,0 & $-1,0$ \\
\hline \multirow{5}{*}{$\begin{array}{l}\text { Casals, } \\
1992\end{array}$} & \multirow[t]{5}{*}{$\mathrm{MN}^{2}$} & \multirow[t]{5}{*}{ Allaitement } & \multirow[t]{5}{*}{15} & \multirow[t]{5}{*}{1000} & 0 & 0 & 1531 & - & 72,1 & - & 50,0 & - \\
\hline & & & & & 50 & 5 & 1497 & -34 & 77,3 & 5,2 & 48,6 & $-1,4$ \\
\hline & & & & & 100 & 10 & 1742 & 211 & 93,2 & 21,1 & 48,9 & $-1,1$ \\
\hline & & & & & 150 & 15 & 1677 & 146 & 86,6 & 14,5 & 49,6 & $-0,4$ \\
\hline & & & & & 200 & 20 & 1623 & 92 & 104,4 & 32,3 & 49,4 & $-0,6$ \\
\hline \multirow{4}{*}{$\begin{array}{l}\text { Casals } \\
\text { et coll., } \\
1999\end{array}$} & \multirow[t]{4}{*}{$\mathrm{MN}$} & Allaitement & 15 & 800 & 0 & 0 & 1631 & - & 73,9 & - & 52,2 & - \\
\hline & & & & & 160 & 20 & 1566 & -65 & 96,6 & 22,7 & 50,2 & $-2,0$ \\
\hline & & & & & 0 & 0 & 1807 & - & 74,1 & - & 51,4 & - \\
\hline & & & & & 160 & 20 & 1530 & -277 & 103,5 & 29,4 & 49,8 & $-1,6$ \\
\hline Espinoza & $\mathrm{PB}^{3}$ & Allaitement & 60 & 900 & 0 & 0 & - & - & 32,0 & - & 53,0 & - \\
\hline et coll., & & & & & 50 & 5,5 & & & 34,0 & 2,0 & 53,0 & 0,0 \\
\hline & & & 60 & 900 & 0 & 0 & - & - & 33,0 & - & 50,0 & - \\
\hline & & & & & 50 & 5,5 & & & 50,0 & 17,0 & 49,0 & $-1,0$ \\
\hline Horton & $\mathrm{DT}^{4}$ & Allaitement & 21 & 1000 & 0 & 0 & 2325 & - & 37,9 & & 42,5 & - \\
\hline et coll., & & & & & 150 & 15 & 2236 & -89 & 52,8 & 14,9 & 39,9 & $-2,6$ \\
\hline & & & 21 & 1000 & 0 & 0 & 1879 & - & 38,2 & & 50,2 & - \\
\hline & & & & & 150 & 15 & 1823 & -56 & 53,5 & 15,3 & 48,2 & $-2,0$ \\
\hline & & & 28 & 1000 & 0 & 0 & 2355 & - & - & & 46,9 & - \\
\hline & & & & & 75 & 7,5 & 2325 & -30 & - & - & 43,9 & $-3,0$ \\
\hline & & & & & 150 & 15 & 2203 & -152 & - & - & 46,7 & $-0,2$ \\
\hline & & & & & 300 & 30 & 2191 & -164 & - & - & 38,8 & $-8,1$ \\
\hline & & & 56 & 1000 & 0 & 0 & 1980 & - & 49,8 & - & 54,7 & - \\
\hline & & & & & 75 & 7,5 & 1937 & -43 & 56,6 & 6,8 & 52,1 & $-2,6$ \\
\hline & & & & & 150 & 15 & 1827 & -153 & 55,8 & 6,0 & 52,8 & $-1,9$ \\
\hline & & & & & 300 & 30 & 1442 & -538 & 57,2 & 7,4 & 45,0 & $-9,7$ \\
\hline Pérez Alba & $\mathrm{MN}$ & Allaitement & 24 & 870 & 0 & 0 & 1950 & - & 73,0 & - & 44,0 & - \\
\hline et coll., 1997 & & & & & 145 & 16,6 & 1860 & -90 & 82,0 & 9,0 & 43,6 & $-0,4$ \\
\hline Pérez- & $\mathrm{FD}^{5}$ & Allaitement & 15 & - & 0 & 0 & 2500 & - & 67,0 & - & - & - \\
\hline Hernández & & & & & 250 & - & 2590 & 90 & 93,0 & 26,0 & & \\
\hline & & & & & 0 & 0 & 2500 & - & 67,0 & - & - & - \\
\hline & & & & & 250 & - & 2170 & -330 & 93,0 & 26,0 & & \\
\hline & & & 15 & - & 0 & 0 & 3222 & - & 77,3 & - & - & - \\
\hline & & & & & 75 & - & 3260 & 38 & 88,7 & 11,4 & & \\
\hline & & & & & 150 & - & 3400 & 178 & 99,3 & 22,0 & & - \\
\hline & & & & & 0 & 0 & 3680 & - & 78,0 & - & - & - \\
\hline & & & & & 75 & - & 3770 & 90 & 79,0 & 1,0 & & \\
\hline & & & & & 150 & - & 3790 & 110 & 88,0 & 10,0 & & \\
\hline
\end{tabular}


Tableau I (suite)

\begin{tabular}{|c|c|c|c|c|c|c|c|c|c|c|c|c|}
\hline \multirow[t]{2}{*}{ Référence } & \multirow[t]{2}{*}{ Race } & \multirow[t]{2}{*}{ Etat } & \multirow{2}{*}{$\begin{array}{l}\text { SL } \\
\text { (j) }\end{array}$} & \multirow{2}{*}{$\begin{array}{c}\text { Conc. } \\
(\mathrm{g} / \mathrm{j})\end{array}$} & \multicolumn{2}{|c|}{ Scag } & \multicolumn{2}{|c|}{ Production } & \multicolumn{4}{|c|}{ Composition } \\
\hline & & & & & $(g / j)$ & $\% \mathrm{CC}$ & Lait (ml/j) & $\Delta$ Lait & TB (g/l) & $\Delta \mathrm{TB}$ & TP (g/l) & $\Delta \mathrm{TP}$ \\
\hline \multirow[t]{4}{*}{ Casals, 1992} & \multirow[t]{4}{*}{$\mathrm{MN}$} & \multirow[t]{4}{*}{ Traite } & \multirow[t]{4}{*}{46} & \multirow[t]{4}{*}{800} & 0 & 0 & 1057 & - & 57,9 & - & 55,9 & - \\
\hline & & & & & 160 & 20 & 1046 & -11 & 84,6 & 26,7 & 52,7 & $-3,2$ \\
\hline & & & & & 0 & 0 & 1051 & - & 61,7 & - & 57,0 & - \\
\hline & & & & & 160 & 20 & 860 & -191 & 82,2 & 20,5 & 54,5 & $-2,5$ \\
\hline \multirow[t]{5}{*}{ Casals, 1992} & \multirow[t]{5}{*}{$\mathrm{MN}$} & \multirow[t]{5}{*}{ Traite } & \multirow[t]{5}{*}{46} & \multirow[t]{5}{*}{1000} & 0 & 0 & 979 & - & 71,6 & - & 53,0 & - \\
\hline & & & & & 50 & 5 & 979 & 0 & 80,1 & 8,5 & 51,7 & $-1,3$ \\
\hline & & & & & 100 & 10 & 917 & -62 & 91,9 & 20,3 & 54,4 & 1,4 \\
\hline & & & & & 150 & 15 & 969 & -10 & 84,8 & 13,2 & 50,4 & $-2,6$ \\
\hline & & & & & 200 & 20 & 926 & -53 & 92,0 & 20,4 & 52,3 & $-0,7$ \\
\hline \multirow{5}{*}{$\begin{array}{l}\text { Casals, } \\
1992\end{array}$} & \multirow[t]{5}{*}{$\mathrm{MN}$} & \multirow[t]{5}{*}{ Traite } & \multirow[t]{5}{*}{77} & 800 & 0 & 0 & 820 & - & 71,8 & - & 58,1 & - \\
\hline & & & & & 40 & 5 & 948 & 128 & 78,3 & 6,5 & 56,7 & $-1,4$ \\
\hline & & & & & 80 & 10 & 756 & -64 & 88,5 & 16,7 & 57,6 & $-0,5$ \\
\hline & & & & & 120 & 15 & 851 & 31 & 86,9 & 15,1 & 53,7 & $-4,4$ \\
\hline & & & & & 160 & 20 & 781 & -39 & 91,2 & 19,4 & 54,5 & $-3,6$ \\
\hline Casals, & $\mathrm{MN}$ & Traite & 81 & 600 & 0 & 0 & 782 & - & 74,0 & - & 60,2 & - \\
\hline 1992 & & & & & 120 & 20 & 840 & 58 & 95,9 & 21,9 & 55,6 & $-4,6$ \\
\hline & & & & & 0 & 0 & 738 & - & 79,5 & - & 63,0 & - \\
\hline & & & & & 120 & 20 & 694 & -44 & 100,1 & 20,6 & 59,3 & $-3,7$ \\
\hline Casals, & MN & Traite & 126 & 600 & 0 & 0 & 545 & - & 96,3 & - & 68,0 & - \\
\hline 1992 & & & & & 120 & 20 & 576 & 31 & 104,4 & 8,1 & 58,0 & -10 \\
\hline & $\mathrm{MN}$ & Traite & 126 & 600 & 0 & 0 & 535 & - & 103,4 & - & 69,1 & - \\
\hline & & & & & 120 & 20 & 540 & 5 & 109,5 & 6,1 & 60,5 & $-8,6$ \\
\hline Casals, & $\mathrm{MN}$ & Traite & 126 & 600 & 0 & 0 & 574 & - & 77,4 & - & 63,7 & - \\
\hline 1992 & & & & & 30 & 5 & 655 & 81 & 87,1 & 9,7 & 65,0 & 1,3 \\
\hline & & & & & 60 & 10 & 541 & -33 & 99,0 & 21,6 & 64,3 & 0,6 \\
\hline & & & & & 90 & 15 & 540 & -34 & 91,2 & 13,8 & 58,7 & $-5,0$ \\
\hline & & & & & 120 & 20 & 557 & -17 & 96,5 & 19,1 & 59,7 & $-4,0$ \\
\hline Cuartero et & $\mathrm{MN}$ & Traite & 100 & - & 0 & 0 & 451 & - & 92,0 & - & - & - \\
\hline coll., 1992 & & & & & 75 & - & 461 & 10 & 104,0 & 12 & & \\
\hline Font, 1991 & $\mathrm{MN}$ & Traite & 98 & 600 & 0 & 0 & 505 & - & 99,7 & - & 64,8 & - \\
\hline & & & & & 72 & 12 & 529 & 24 & 105,0 & 5,3 & 61,0 & $-3,8$ \\
\hline & & & 126 & 600 & 0 & 0 & 445 & - & 102,9 & - & 67,0 & - \\
\hline & & & & & 72 & 12 & 481 & 36 & 113,0 & 10,1 & 65,6 & $-1,4$ \\
\hline McKusick et & $\mathrm{FE}^{6}$ & Traite & 40 & - & 0 & 0 & 2970 & - & 44,9 & - & 54,2 & - \\
\hline coll., 1999 & & & & & 100 & - & 2870 & -100 & 56,6 & 11,7 & 49,9 & $-4,3$ \\
\hline Pérez Alba & $\mathrm{MN}$ & Traite & 53 & 1200 & 0 & 0 & 1400 & - & 64,9 & - & 51,4 & - \\
\hline et coll., 1997 & & & & & 200 & 16,6 & 1560 & 160 & 68,1 & 3,2 & 48,9 & $-2,5$ \\
\hline Rotunno & $\mathrm{CN}^{7}$ & Traite & 110 & 910 & 0 & 0 & - & - & 89,8 & - & 63,9 & - \\
\hline et coll., & & & & & 36 & 4 & - & - & 88,0 & $-1,8$ & 59,2 & $-4,7$ \\
\hline 1998 & & & & & 72 & 8 & - & - & 92,1 & 2,3 & 59,8 & $-4,1$ \\
\hline
\end{tabular}

${ }^{1}$ Polypay $;{ }^{2}$ Manchega $;{ }^{3}$ Pelibuey $;{ }^{4}$ Dorset $;{ }^{5}$ Finn Dorset $;{ }^{6}$ Frisonne de 1 'Est $;{ }^{7}$ Comisana 


\section{Traitement des données et analyses statistiques}

L'ensemble des données de la base bibliographique a été traité à partir du logiciel Spss (version 13) spécialisé dans les régressions. Un premier type de modèle n'a pris en compte que l'effet de la dose de lipides protégés pour expliquer les réponses de chaque variable. Afin d'améliorer la précision du modèle, ont été intégrées ensuite les autres variables, telles que le stade de lactation, la production initiale, le TB initial et le TP initial qui semblaient expliquer une partie importante des variations de la réponse. Plusieurs types de régressions ont été analysées (simples, polynomiales, multiples); les plus significatives $(\mathrm{P}<0,05)$ ont été retenues, ainsi que celles qui présentaient le coefficient de détermination $\left(\mathrm{R}^{2}\right)$ le plus élevé.

\section{RESULTATS ET DISCUSSION}

Comme pour les vaches laitières, la réponse à l'incorporation des lipides protégés sur les paramètres productifs des brebis est variable en conditions expérimentales. Dans ce qui suit, l'auteur a essayé d'étudier les réponses en fonction des quantités de lipides protégés ingérées, du stade de lactation et des TB et TP initiaux. Dans cette analyse, il n'a pas été possible d'aborder les effets des composants de la ration de base sur ces réponses par manque de données chiffrées dans les publications. Dans le tableau II, sont récapitulées les caractéristiques des variables et des paramètres analysés. Dans ces études, les TB et TP moyens des laits d'allaitement ont été supérieurs à ceux de la traite, inversement à la production laitière brute. Afin de consolider la précision des régressions, les données des phases d'allaitement et de traite ont été rassemblées pour être analysées conjointement.

\section{Production laitière}

Les réponses de la production laitière brute suite à l'incorporation des Scag dans la ration des brebis sont relativement variables et présentent, comme pour le cas des vaches laitières, autant de cas positifs (43 p. 100) que négatifs (57 p. 100, tableau I). En moyenne brute, l'effet est faible et négatif (- $25 \mathrm{ml} / \mathrm{j})$. Selon Casals (5), l'absence de réponse de production de lait peut être attribuée à la variabilité individuelle et au faible nombre de brebis disponibles par étude. D'autre part, il est possible que le potentiel laitier des brebis utilisées soit insuffisant pour mettre en évidence un effet positif des lipides protégés sur le bilan énergétique comme pour le cas des vaches laitières (10).

\section{Effet du niveau des apports}

L'analyse des résultats du tableau I a permis d'établir une relation curvilinéaire entre la variation de la production laitière (PL) brute et le niveau quantitatif des apports (figure 1) :

$$
\begin{aligned}
& \Delta \mathrm{PL}(\mathrm{ml} / \mathrm{j})=0,6568 \cdot \mathrm{Scag}(\mathrm{g} / \mathrm{j})-0,0053 \cdot \mathrm{Scag}^{2}(\mathrm{~g} / \mathrm{j}) \\
& \left(\mathrm{n}=46, \mathrm{R}^{2}=0,258, \mathrm{P}<0,01\right)[1] \\
& ( \pm 0,3414, \mathrm{P}>0,05)( \pm 0,0017, \mathrm{P}<0,01)
\end{aligned}
$$

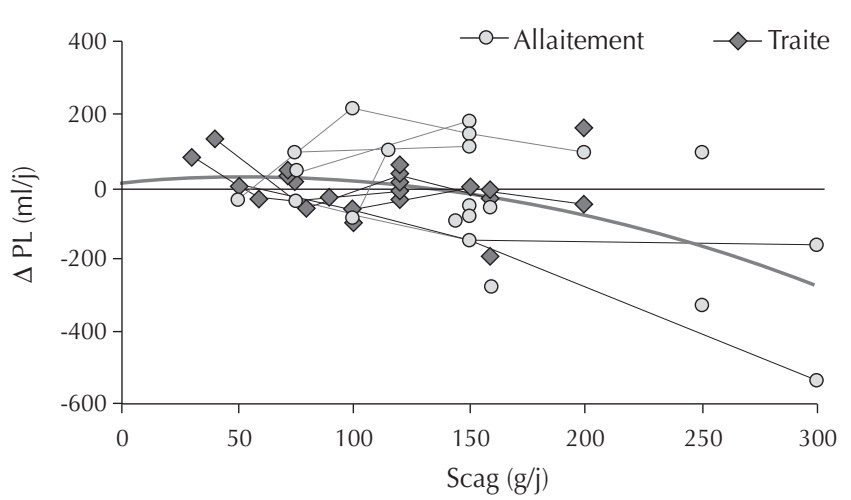

Figure 1 : variation de la production laitière $(P L)$ brute en fonction de la quantité de savons de calcium d'acides gras (Scag) apportée dans la ration.

\section{Tableau II}

Caractéristiques des variables analysées dans les bases bibliographiques

Variable

\begin{tabular}{|c|c|c|c|c|c|c|c|c|c|c|}
\hline & & & & & & & & & & \\
\hline & $\mathbf{n}$ & Moyenne & ET & Min & Max & $\mathbf{n}$ & Moyenne & ET & Min & $\operatorname{Max}$ \\
\hline Scag (g/j) & 25 & 143 & 72,2 & 50 & 300 & 25 & 105 & 48,0 & 30 & 200 \\
\hline Stade de lactation (j) & 25 & 27 & 16,5 & 15 & 60 & 25 & 86 & 32,8 & 40 & 126 \\
\hline Production lait témoin & 23 & 2271 & 650 & 1531 & 3680 & 23 & 868 & 518 & 445 & 2970 \\
\hline Production lait exp. & 23 & 2225 & 706 & 1442 & 3790 & 23 & 864 & 504 & 461 & 2870 \\
\hline$\Delta$ lait & 23 & -46 & 176 & -538 & 211 & 23 & -4 & 74 & -191 & 160 \\
\hline TB initial & 22 & 64,8 & 18,0 & 32,0 & 99,0 & 25 & 77,6 & 14,2 & 44,9 & 103,4 \\
\hline TB expérimental & 22 & 80,9 & 23,3 & 34,0 & 121,0 & 25 & 90,8 & 12,5 & 56,6 & 113 \\
\hline$\Delta \mathrm{TB}(\mathrm{g} / \mathrm{l})$ & 22 & 16,0 & 9,6 & 1,0 & 32,3 & 25 & 13,2 & 7,4 & $-1,8$ & 26,7 \\
\hline TP initial & 19 & 49,6 & 3,5 & 42,5 & 54,7 & 24 & 59,9 & 5,3 & 51,4 & 69,1 \\
\hline TP expérimental & 19 & 47,4 & 3,9 & 38,8 & 53,0 & 24 & 56,8 & 4,7 & 48,9 & 65,6 \\
\hline$\Delta \mathrm{TP}(\mathrm{g} / \mathrm{l})$ & 19 & $-2,1$ & 2,5 & $-9,7$ & 0 & 24 & $-3,1$ & 2,7 & $-10,0$ & 1,4 \\
\hline
\end{tabular}

Période d'allaitement

Période de traite

Scag : savons de calcium d'acides gras

$\Delta$ lait : variation de la production $; \Delta \mathrm{TB}$ : variation du taux butyreux ; $\Delta \mathrm{TP}$ : variation du taux protéique

$\mathrm{n}$ : nombre de données disponibles; ET : écart-type de la variable

Min : valeur minimale de la variable ; Max : valeur maximale de la variable 
De cette relation, on peut conclure que les lipides protégés n'ont pas d'effet sur la production laitière des brebis lorsqu'ils sont apportés à des doses modérées (au-dessous de $100 \mathrm{~g} / \mathrm{j}$ ) ou quand les lipides ajoutés représentent 3-5 p. 100 de la ration (tableau I). En revanche, les réponses négatives sont observées avec des doses plus élevées (au-delà de $150 \mathrm{~g} / \mathrm{j}$, figure 1) ou quand le supplément représente plus de 6 p. 100 de la matière sèche de la ration. Dans la plupart des cas, la baisse de la production laitière est principalement attribuée à la diminution de consommation d'énergie par les animaux supplémentés $(19,21,24)$. Dans certaines études où la consommation d'énergie est accrue par l'apport de fortes quantités de Scag (supérieures à $160 \mathrm{~g} / \mathrm{j}$ ), la production laitière a même été améliorée. Ceci ne fait que confirmer la forte relation qui existe entre la production laitière et la consommation d'énergie $(7,19$, $24,28)$.

\section{Effet du stade de lactation}

L'analyse conjointe des résultats de la bibliographie (tableau I) n'a pas permis d'établir une relation significative entre la variation de la production laitière brute selon le stade de lactation des brebis supplémentées $\left(n=46, R^{2}=0,008, P>0,05\right)$. De même, l'introduction de ce paramètre dans l'équation [1] n'a pas permis d'améliorer la précision :

$\Delta \mathrm{PL}(\mathrm{ml} / \mathrm{j})=0,8489 \cdot \mathrm{Scag}(\mathrm{g} / \mathrm{j})-0,0059 \cdot \mathrm{Scag}^{2}(\mathrm{~g} / \mathrm{j})-0,2380 \bullet \mathrm{SL}(\mathrm{j})$ $\left(\mathrm{n}=46, \mathrm{R}^{2}=0,264, \mathrm{P}<0,01\right)[2]$

$( \pm 0,4765, \mathrm{P}>0,05)( \pm 0,0020, \mathrm{P}<0,01)( \pm 0,4084, \mathrm{P}>0,05)$

L'absence de réponse de production de lait brute a été mentionnée, que ce soit durant la période d'allaitement $(1,7,25)$ ou durant la traite $(6,8)$.

\section{Taux butyreux du lait}

Dans la plupart des références bibliographiques disponibles, on signale un effet positif des Scag sur le TB du lait de brebis $(4,7$, 29). Cependant, il faut signaler que cette réponse dépend aussi du taux d'incorporation de ces lipides, des constituants de la ration, du niveau de production et du stade de lactation des brebis et de leur état nutritionnel $(3,4)$.

\section{Effet du niveau des apports}

Une grande partie de la variabilité de la réponse du TB du lait peut être reliée à la dose de lipides protégés ingérée. Selon Casals et coll. (6), et Schmidely et Sauvant (29), il existe généralement une relation curvilinéaire entre ces deux paramètres. En effet, l'analyse des résultats du tableau I confirme ces observations (figure 2) :

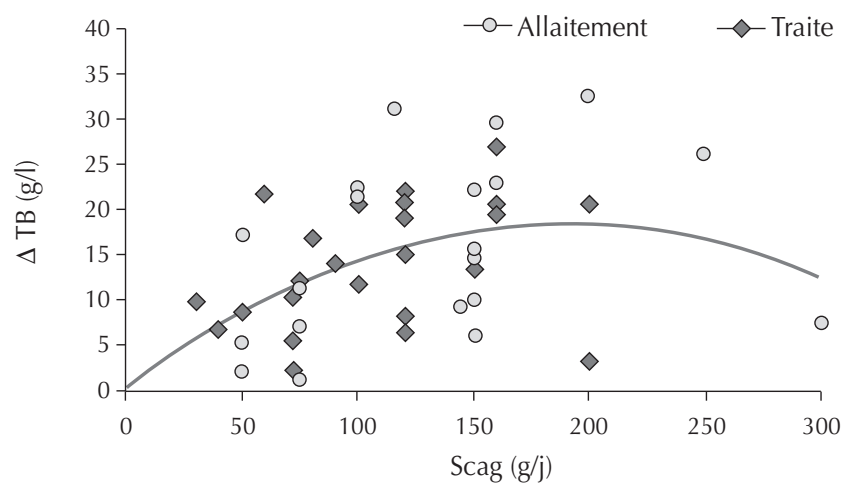

Figure 2 : variation du taux butyreux (TB) du lait en fonction de la quantité de savons de calcium d'acides gras (Scag) selon le stade physiologique des brebis.
$\Delta \mathrm{TB}(\mathrm{g} / \mathrm{l})=0,1904 \cdot \mathrm{Scag}(\mathrm{g} / \mathrm{j})-0,0005 \cdot \mathrm{Scag}^{2}(\mathrm{~g} / \mathrm{j})$

$\left(\mathrm{n}=47, \mathrm{R}^{2}=0,276, \mathrm{P}<0,001\right)[3]$

$( \pm 0,0228, \mathrm{P}<0,001)( \pm 0,0001, \mathrm{P}<0,001)$

Selon cette équation, la réponse maximale chez la brebis $(+18 \mathrm{~g} / \mathrm{l})$ est ainsi atteinte dès l'apport de $190 \mathrm{~g} / \mathrm{j}$ de Scag (dérivée de l'équation [2] = 0). Ces valeurs sont un peu plus élevées que celles rapportées par Bocquier et Caja (3), et Schmidely et Sauvant (29) mais comparables à celles de Casals (5), et Horton et coll. (21). Selon Casals (5), et Casals et coll. (6), le meilleur effet sur le TB du lait est obtenu quand le niveau d'inclusion des lipides protégés apporte à peu près 10 p. 100 de l'énergie consommée. Cependant, quand le niveau d'apport est très élevé (> $250 \mathrm{~g} / \mathrm{j} / \mathrm{brebis})$, le TB du lait commence à diminuer, indiquant l'existence d'un effet de saturation de la réponse (figure 2).

La production totale de matières grasses évolue aussi de forme quadratique avec le niveau d'apport :

$\Delta \operatorname{MGT}(\mathrm{g} / \mathrm{j})=0,3033 \cdot \mathrm{Scag}(\mathrm{g} / \mathrm{j})-0,0008 \cdot \mathrm{Scag}^{2}(\mathrm{~g} / \mathrm{j})$

$\left(\mathrm{n}=43, \mathrm{R}^{2}=0,102, \mathrm{P}<0,05\right)[4]$

$( \pm 0,0677, \mathrm{P}<0,001)( \pm 0,0004, \mathrm{P}<0,05)$

De cette relation, le meilleur rendement peut être obtenu avec un apport journalier de Scag proche aussi de $190 \mathrm{~g} / \mathrm{j}$ (dérivée de l'équation $[3]=0)$.

Ces résultats ainsi que ceux concernant la production laitière et le TB montrent qu'il serait possible d'optimiser la quantité de Scag apportée en fonction, d'une part, du coût économique de ces matières premières et, d'autre part, des objectifs zootechniques souhaités : accroître la production laitière $(\mathrm{Scag}<120 \mathrm{~g} / \mathrm{j})$ ou le TB et la MG sécrétée $(\mathrm{Scag} \approx 200 \mathrm{~g} / \mathrm{j})$.

\section{Effet du stade de lactation}

Sur la figure 3 est représentée l'évolution de la réponse du TB du lait à l'incorporation des Scag en fonction du stade de lactation. La relation suivante a pu être établie:

$\Delta \mathrm{TB}(\mathrm{g} / \mathrm{l})=18,77-0,0728 \cdot \mathrm{SL}(\mathrm{j})$

$\left(\mathrm{n}=47, \mathrm{R}^{2}=0,116, \mathrm{P}<0,05\right)[5]$

$( \pm 2,09, \mathrm{P}<0,001)( \pm 0,0299, \mathrm{P}<0,05)$

Selon cette relation, il en résulte que les réponses les plus marquées sont obtenues en début de lactation chez la brebis, tandis que les essais de milieu ou de fin de lactation induisent des réponses faibles. Ces résultats confirment ceux obtenus antérieurement par Casals et coll. (8), et Perez Alba et coll. (24). Selon ces auteurs, le meilleur

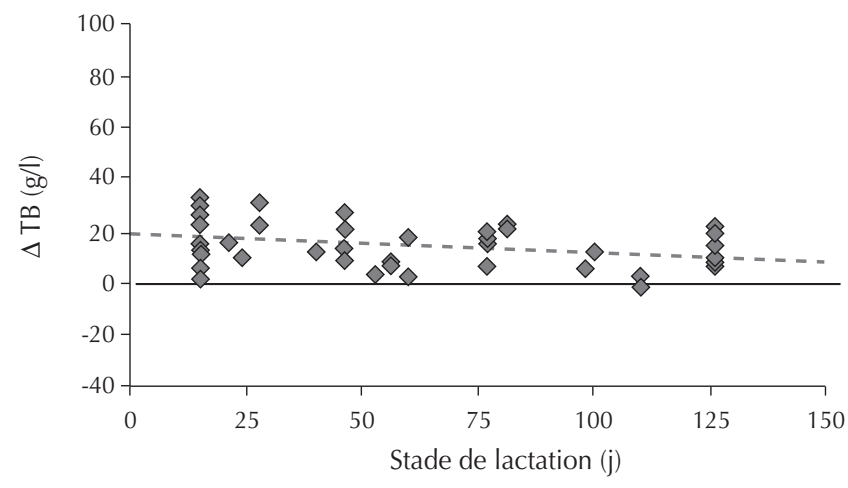

Figure 3 : réponse du taux butyreux $(T B)$ du lait à l'apport de savons de calcium d'acides gras (Scag) selon le stade de lactation. 
rendement est obtenu durant la période d'allaitement (0-5 semaines de lactation), avec un accroissement de $15 \mathrm{~g}$ de TB/l pour chaque $100 \mathrm{~g}$ de Scag apporté dans le concentré. Ces réponses peuvent s'interpréter comme une meilleure efficacité du transfert des acides gras (AG) alimentaires vers la mamelle en début de lactation et/ou une plus grande efficacité de leur transfert vers le tissu adipeux en milieu de lactation afin de reconstituer les réserves corporelles (11).

Dans la plupart des études, les compositions des rations et les caractéristiques des animaux (poids vif, variation de poids), souvent faiblement renseignées, n'ont pas permis de calculer le bilan énergétique des animaux sur un nombre suffisant d'essais. Cependant, en considérant les variations de poids ou les niveaux d'ingestion pour ces essais en début de lactation, les réponses les plus faibles du TB et de la matière grasse sont obtenues lorsque les apports énergétiques sont les plus élevés (25) ou lorsque les variations de poids sont les plus faibles $(21,24)$. Ces résultats couplés à la faible réponse en milieu de lactation suggèrent que, chez la brebis, le bilan énergétique module l'efficacité de l'utilisation des matières grasses alimentaires, probablement au travers de la modulation des activités respectives de la lipoprotéine lipase dans la mamelle et le tissu adipeux (29).

Enfin, il faut signaler l'absence d'interaction significative entre le stade de lactation et la quantité de Scag chez la brebis. Selon l'étude de Schmidely et Sauvant (29), la variabilité de ces réponses peut être attribuée à différents facteurs, comme le niveau de production laitière, le TB du lait des animaux témoins ou le bilan énergétique. Dans cette étude, la considération du TB initial (TBi) et de la PL initiale (PLi) n'a pas amélioré la précision du modèle, indiquant leur faible incidence sur la variabilité de la réponse du TB :

$\Delta \mathrm{TB}(\mathrm{g} / \mathrm{l})=0,2001 \cdot \mathrm{Scag}(\mathrm{g} / \mathrm{j})-0,0005 \cdot \mathrm{Scag}^{2}(\mathrm{~g} / \mathrm{j})-0,0091 \cdot \mathrm{TBi}(\mathrm{g} / \mathrm{l})$ $\left(\mathrm{n}=47, \mathrm{R}^{2}=0,277, \mathrm{P}<0,01\right)[6]$

$( \pm 0,0534, \mathrm{P}<0,001)( \pm 0,0002, \mathrm{P}<0,05)( \pm 0,0450, \mathrm{P}>0,05)$

$\Delta \mathrm{TB}(\mathrm{g} / \mathrm{l})=0,2097 \cdot \mathrm{Scag}(\mathrm{g} / \mathrm{j})-0,0005 \cdot \mathrm{Scag}^{2}(\mathrm{~g} / \mathrm{j})-0,0008 \cdot \mathrm{PLi}(\mathrm{ml} / \mathrm{j})$ $\left(\mathrm{n}=43, \mathrm{R}^{2}=0,217, \mathrm{P}<0,05\right)[7]$

$( \pm 0,0319, \mathrm{P}<0,001)( \pm 0,0001, \mathrm{P}<0,001)( \pm 0,0012, \mathrm{P}>0,05)$

\section{Taux protéique du lait}

Comme pour le cas des vaches laitières, les Scag affectent négativement le TP du lait de brebis (tableau I). Les causes possibles de cette diminution sont discutées dans plusieurs revues consacrées aux vaches laitières $(10,23)$. Selon les résultats du tableau I, la réponse dépend de la dose de lipides et du stade de lactation.

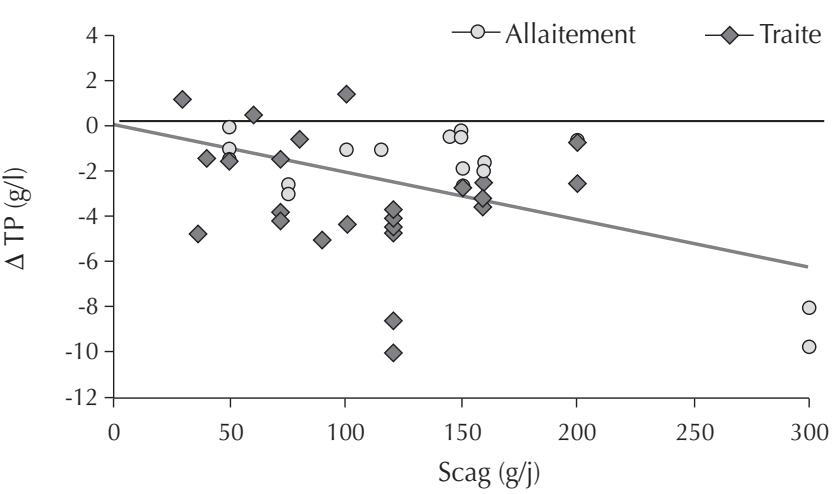

Figure 4 : variation du taux protéique $(T P)$ du lait en fonction de la quantité de savons de calcium d'acides gras (Scag) selon le stade physiologique des brebis.

\section{Effet du niveau des apports}

Sur la figure 4 est représentée la variation du TP du lait en fonction des quantités de Scag consommées par les brebis. La relation obtenue est la suivante :

$\Delta \mathrm{TP}(\mathrm{g} / \mathrm{l})=-0,0210 \cdot \mathrm{Scag}(\mathrm{g} / \mathrm{j})$

$\left(\mathrm{n}=43, \mathrm{R}^{2}=0,144, \mathrm{P}<0,001\right)[8]$

$( \pm 0,0028, \mathrm{P}<0,001)$

Il existe donc une relation linéaire et négative entre le TP du lait et le niveau d'ingestion de Scag chez les ovins laitiers. C'est avec des doses modérées (inférieures à $100 \mathrm{~g} / \mathrm{j}$ ) que la diminution du TP du lait de brebis est moins importante. En revanche, les réponses les plus négatives sont observées avec une ingestion de Scag supérieure à $150 \mathrm{~g} / \mathrm{j}$ (figure 4).

En tenant compte de la réponse de la quantité totale de matières protéiques du lait avec l'ingestion de Scag, on obtient la relation suivante :

$\Delta$ MPT $(g / j)=0,0236 \cdot S c a g(g / j)-0,0004 \cdot \operatorname{Scag}^{2}(g / j)$

$\left(\mathrm{n}=39, \mathrm{R}^{2}=0,458, \mathrm{P}<0,001\right)[9]$

$( \pm 0,0215, \mathrm{P}>0,05)( \pm 0,0001, \mathrm{P}<0,001)$

De cette relation, on peut considérer que le rendement protéique n'est pas affecté par l'apport des Scag jusqu'à des valeurs d'ingestion supérieures à $50 \mathrm{~g} / \mathrm{j}$.

\section{Effet du stade de lactation}

L'analyse des résultats du tableau I permet d'obtenir la relation suivante :

$$
\begin{aligned}
& \Delta \mathrm{TP}(\mathrm{g} / \mathrm{l})=-1,30-0,0221 \cdot \mathrm{SL}(\mathrm{j}) \\
& \left(\mathrm{n}=43, \mathrm{R}^{2}=0,104, \mathrm{P}<0,05\right)[10] \\
& ( \pm 0,730, \mathrm{P}>0,05)( \pm 0,0102, \mathrm{P}<0,05)
\end{aligned}
$$

L'effet négatif des lipides protégés sur le TP ne se manifeste généralement qu'en pleine lactation chez les brebis laitières (figure 5). Il ressort donc, comme pour le cas des vaches laitières $(9,20)$, que le stade physiologique de la brebis joue un rôle important sur la réponse du TP aux apports lipidiques. Ces observations ont été aussi signalées par Casals et coll. (8), et Espinoza et coll. (13), qui n'ont pas observé de diminution de la teneur en protéines du lait durant les périodes d'allaitement et de début de lactation. A partir de la seconde moitié de lactation, ces auteurs ont observé un effet négatif de type linéaire $(-2,5 \mathrm{~g} / \mathrm{l}$ de lait pour chaque $100 \mathrm{~g}$ de Scag) qui a été plus évident en fin de lactation.

En tenant compte de la dose et du stade de lactation ensemble, on peut établir la relation suivante :

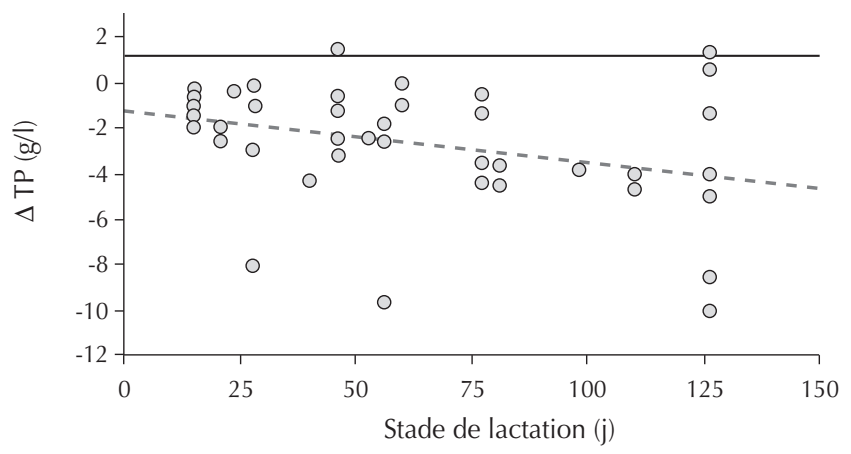

Figure 5 : réponse du taux protéique (TP) du lait à l'apport de savons de calcium d'acides gras selon le stade de lactation. 
$\Delta \mathrm{TP}(\mathrm{g} / \mathrm{l})=-0,0138 \cdot \mathrm{Scag}(\mathrm{g} / \mathrm{j})-0,0203 \cdot \mathrm{SL}(\mathrm{j})$

$\left(\mathrm{n}=43, \mathrm{R}^{2}=0,318, \mathrm{P}<0,001\right)[11]$

$( \pm 0,0033, \mathrm{P}<0,001)( \pm 0,0063, \mathrm{P}<0,01)$

De cette relation, on peut conclure que la réponse du TP du lait à l'incorporation des Scag est fonction du niveau des apports et du stade de lactation.

Finalement, on doit signaler que les essais pour éviter la dépression protéique due aux lipides alimentaires ont été peu étudiés chez les ovins laitiers. On peut citer ceux de Casals et coll. (7), et Goulas et coll. (19) qui ont étudié l'effet d'une augmentation de la proportion de protéines non dégradables dans la ration supplémentée avec des Scag. Cette dépression n'a pu être que partiellement réduite durant la période centrale de lactation.

\section{Vitesse de croissance des agneaux}

Les études concernant l'utilisation des lipides protégés dans la ration des brebis allaitantes sont peu nombreuses $(1,7,13,16$, 24, 25). En général, l'amélioration de la vitesse de croissance des agneaux des brebis supplémentées est faible et parfois nulle malgré l'augmentation du contenu énergétique du lait consommé par les agneaux. Casals (5) explique cette faible réponse par une possible réduction de la digestibilité de certains $\mathrm{AG}$ à chaîne longue du lait (par ex. palmitique et oléique) chez les jeunes agneaux. D'autre part, il faut signaler que le profil des AG du lait consommé par ces agneaux est modifié par les apports lipidiques, avec notamment une diminution des $\mathrm{AG}$ à chaîne courte et moyenne $(18,24)$. Or, il est connu que les jeunes agneaux digèrent mieux les AG courts que longs (2), ce qui peut expliquer en partie la faible amélioration de leur vitesse de croissance.

Gargouri (16), et Sanz Sampelayo et coll. (27) signalent que le facteur limitant pour la croissance des préruminants est l'ingestion de protéines et non pas d'énergie. La diminution du TP du lait, observée dans la plupart des cas avec l'emploi des Scag, peut expliquer aussi l'absence de réponse chez les agneaux.

\section{Composition en acides gras du lait}

Les matières grasses du lait des ruminants se caractérisent par leur contenu élevé en AG saturés comme conséquence de l'hydrogénation ruminale des AG polyinsaturés. L'un des buts de l'incorporation des lipides protégés dans la ration des ruminants est de pouvoir manipuler la composition des AG du lait en vue d'augmenter la teneur en $A G$ insaturés pour en faire un produit qui répond mieux aux critères technologiques, nutritionnels, organoleptiques et sanitaires des matières grasses laitières (29).

Les matières grasses qui échappent aux modifications du rumen représentent un outil efficace pour modifier la nature et les proportions relatives des acides gras de la matière grasse du lait. Etant donné que ces lipides sont protégés contre l'hydrolyse et l'hydrogénation au niveau du rumen, leur introduction dans la ration provoque généralement une augmentation du pourcentage des AG de même longueur de chaîne que ceux ajoutés au régime. Compte tenu de la richesse en AG insaturés à 18 atomes de carbone de la plupart de ces lipides, les proportions de la matière grasse du lait dans ces AG et dans leurs isomères sont généralement augmentées. C'est ainsi que l'incorporation des savons de calcium riches en acides palmitique $(\mathrm{C} 16: 0)$ et oléique $(\mathrm{C} 18: 1)$ peut améliorer de 14 à 20 p. 100 la proportion de ces deux AG dans le lait de brebis (17, 20, 26). En revanche, on assiste à une forte diminution des teneurs en acides gras courts et moyens du lait (figure 6). Comme conséquence de ces modifications, la proportion des acides gras monoinsaturés est accrue au détriment des saturés.

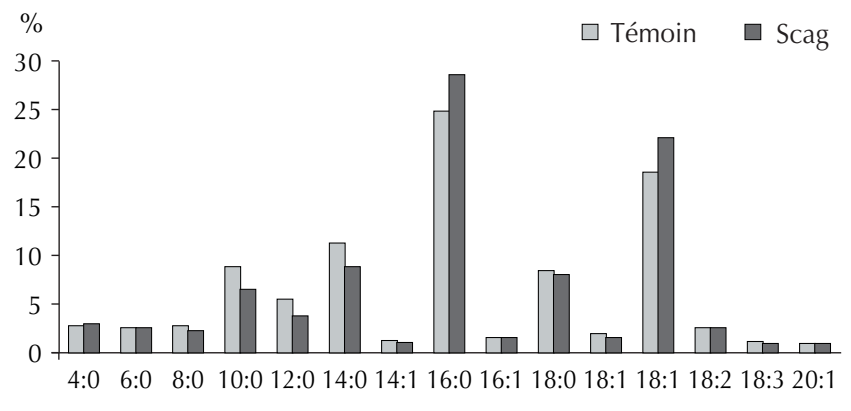

Acides gras

Figure 6 : effet des savons de calcium d'acides gras (Scag) sur la composition en acides gras du lait de brebis (d'après Gargouri et coll., 1997, ITEA Prod. Anim.).

Les effets des suppléments lipidiques sur le profil des AG du lait doivent être pris en considération étant donné les modifications qu'ils peuvent engendrer sur les propriétés physiques ou chimiques de la matière grasse du lait. Cependant, il ne faut pas oublier que les laits riches en $A G$ insaturés peuvent présenter des problèmes d'oxydation et des altérations du goût $(15,30)$.

\section{CONCLUSION}

Les variations des performances des brebis laitières résultant de la complémentation en matières grasses protégées de la ration ont été compilées et la littérature existante sur les savons calciques a été passée en revue. Les résultats montrent que l'utilisation de ce type de supplément n'affecte pas le volume de lait produit mais entraîne en général une augmentation du TB et une diminution du TP du lait. La réponse de ces deux taux dépend de la dose de lipides et du stade de lactation des brebis.

La composition en AG du lait de brebis est presque toujours affectée par les lipides alimentaires, avec notamment une diminution de la proportion des acides gras à chaîne courte et moyenne, et une augmentation des acides gras à chaîne longue. Les lipides protégés qui échappent à la biohydrogénation au niveau du rumen peuvent donc représenter un moyen intéressant pour changer la composition en AG du lait des brebis, que ce soit pour améliorer sa qualité technologique ou diététique.

\section{BIBLIOGRAPHIE}

1. APPEDU L.A., ELY D.G., AARON D.K., DEWEESE W.P., FINK E., 2004 Effects of supplementing with calcium salts of palm oil fatty acids or hydrogenated tallow on ewe milk production and twin lamb growth. J. Anim. Sci., 82: 2780-2789.

2. BAUCHART D., 1981. Digestion comparée des lipides chez les ruminants et les monogastriques. Bull. tech. Crzv Theix, 46 : 45-55.

3. BOCQUIER F., CAJA G., 1993. Recent advances on nutrition and feeding of dairy sheep. In: Proc. 5th Symp. Machine milking of small ruminants, Budapest, Hungary, 14-20 May. Hung. J. Anim. Prod., 1 (Suppl.): 580-607

4. BOCQUIER F., CAJA G., 2001. Productions et composition du lait de brebis : effets de l'alimentation. Prod. Anim., 14 : 129-140.

5. CASALS R., 1992. Efectos de la utilización de lípidos protegidos en la alimentación de ovejas de ordeño durante los periodos de lactación y cubrición. Thèse Doct. vét., UAB, Barcelone, Espagne, 186 p.

6. CASAlS R., CAJA G., GUILLOU D., TORRE C., SUCH X., 1992. Influence of dietary levels of calcium soaps of long chain fatty acids on lactational performance of dairy ewes. J. Dairy Sci., 75: 174.

7. CASALS R., CAJA G., SUCH X., TORRE C., CALSAMIGLIA S., 1999. Lactational effects of calcium soaps and undegraded intake protein on dairy ewes. J. Dairy Res., 66: 177-191. 
8. CASALS R., CAJA G., SUCH X., TORRE C., FABREGAS X., 1992 Lactational evaluation of effects of calcium soaps and undegraded intake protein on dairy ewes. J. Dairy Sci., 75: 174.

9. CHILLIARD Y., DOREAU M., FERLAY A., 1989. Les matières grasses, une source d'énergie pour les vaches laitières. Prod. lait. mod., 190 : 67-68.

10. CHILLIARD Y., GAGLIOSTRO G., FLECHET J., LEFAIVRE J. SEBASTIAN I., 1991. Duodenal rapessed oil infusion in early and midlactation cows. 5. Milk fatty acids and adipose tissue lipogenic activities. J. Dairy Sci., 74: 1844-1854.

11. CHILLIARD Y., DOREAU M., GAGLIOSTRO G., ELMEDDAH Y. 1993. Addition de lipides protégés (encapsulés ou savons de calcium) à la ration de vaches laitières. Effets sur les performances et la composition du lait. Prod. Anim., 6 : 139-150.

12. CUARTERO TJ.R, PEREZ-SEMPERE MATARREDONDA J.I. GOMEZ MARTINEZ V., OTAL SALAVERRY J., 1992. El empleo de la grasa by-pass en la alimentation de la oveja manchega durante el ordeño. Castilla-La-Mancha, España, Conserjería de Agricultura de la Junta de Comunidades, 13 p.

13. ESPINOZA J.L., LOPEZ-MOLINA O., RAMIREZ-GODINEZ J.A. JIMENEZ J., FLORES A., 1998. Milk composition, postpartum reproductive activity and growth of lambs in Pelibuey ewes fed calcium soap of long chain fatty acids. Small Ruminant Res., 27: 119-124.

14. FONT F., 1991. Effets de l'apport de lipides protégés sur la composition du lait de brebis de race Manchega et des fromages. Mémoire DAA, Ensa, Rennes, France, 71 p.

15. FRAGA M.J., PEREZ P., 1987. Utilización de grasa en la alimentación. Bovis, 16: 45-55.

16. GARGOURI A., 1997. Efectos de la utilización de jabones calcicos de acidos grasos de cadena larga en ovejas lecheras durante los periodos de cria y ordeño. Thèse Doct. vét., UAB, Barcelone, Espagne, 174 p.

17. GARGOURI A., CAJA G., GAFO C., SUCH X., FERRET A. 1997. Modificación del perfil de ácidos grasos de la leche de oveja mediante el empleo de jabones cálcicos de ácidos grasos de cadena larga. ITEA Prod. Anim., 18 (suppl.): 694-696.

18. GARGOURI A., CAJA G., SUCH X., CASALS R., FERRET A., 1995. Efectos de la utilización de lípidos protegidos en la alimentación de ovejas de ordeño en el sistema a media leche. ITEA Prod. Anim., 16 (suppl.): 720-722

19. GOULAS C., ZERVAS G., PAPADOPOULOS G., 2003. Effect of dietary animal fat and methionine on dairy ewes milk yield and milk composition. Anim. Feed Sci. Tech., 105: 43-54.

\section{Summary}

Gargouri A. Production and Composition of Ewe's Milk. Effects of Protected Fat Supplementation

A database on the effects of protected fat supplementation [calcium soap of fatty acids (CSFA)] was developed to analyze these effects on milk production and composition in dairy ewes. On the whole, CSFA supplementation does not affect the quantity of milk marketable, it increases milk fat content $(+21 \%)$ and decreases milk protein content $(-4 \%)$. Results greatly vary depending on the quantity of fat supplemented, and the ewe lactation stage. The best responses are observed with an addition of CSFA close to 150 g/day/ewe at the onset of lactation. The composition of milk fatty acids is almost always modified by protected fats, as percentages of short and medium chain fatty acids are reduced, and those of long chain fatty acids are increased. Including CSFA in the concentrate of dairy sheep does not improve lamb growth during the suckling period.

Keywords: Sheep - Ewe milk - Lipids - Chemical composition Lamb - Growth - Tunisia.
20. HOFFMANN P.C., GRUMMER R.R., SHAVER R.D., BRODERICK G.A., DRENDEL T.R., 1991. Feeding supplemental fat and undegraded intake protein to early lactation dairy cows. J. Dairy Sci., 74: 3468-3474.

21. HORTON G.M.J., WOHLT J.E., PALATINI D.D., BALDWIN J.A., 1992. Rumen-protected lipid for lactating ewes and their nursing lambs. Small Ruminant Res., 9: 27-36.

22. MCKUSICK B.C., BERGER Y.M., THOMAS D.L., 1999. Rumenprotected bypass fat for dairy ewe commercial milk production. In: Proc. 5th Great Lakes dairy sheep symposium, Brattleboro, VT, USA, 4-6 Nov.

23. PALMQUIST D.L., WEISBJERG M.R., HVELPLUND T., 1993. Ruminal, intestinal, and total digestibilities of nutrients in cows fed diets high in fat and undegradable protein. J. Dairy Sci., 76: 1353-1364.

24. PEREZ ALBA L.M., DE SOUZA S., PEREZ M., MARTINEZ A., FERNANDEZ G., 1997. Calcium soaps of olive fatty acids in the diets of Manchega dairy ewes: Effects on digestibility and production. J. Dairy Sci., 80: 3316-3324.

25. PEREZ-HERNANDEZ M., ROBINSON J.J., AITIKEN R.P., FRAZER C., 1986. The effect of dietary supplements of protected fat on the yield and fat content of ewe's milk and on lamb growth rate. Anim. Prod., 42: 455.

26. ROTUNNO T., SEVI A., DI CATERINA R., MUSCIO A., 1998. Effects of graded levels of dietary rumen-protected fat on milk characteristics of Comisana ewes. Small Ruminant Res., 30: 137-145.

27. SANZ SAMPELAYO M.R., LARA L., GIL F., BOZA J., 1995. Energy utilization for maintenance and growth in preruminant kid goats and lambs. Small Ruminant Res., 17: 25-30.

28. SANZ SAMPELAYO M.R., PEREZ L., MARTIN ALONSO J.J., AMIGO L., BOZA J., 2002. Effects of concentrates with different contents of protected fat rich in PUFAs on the performance of lactating Granadina. II. Milk production and composition. Small Ruminant Res., 43: 141-148.

29. SCHMIDELY D., SAUVANT D., 2001. Taux butyreux et composition de la matière grasse du lait chez les petits ruminants : effets de l'apport de matières grasses ou d'aliment concentré. Prod. Anim., 14 : 337-354.

30. SIDHU G.S., BROWN M.A., JOHNSON A.R., 1975. Autoxidation in milk rich in linoleic acid. I. An objective method for measuring autoxidation and evaluating antioxidants. J. Dairy Res., 42: 185-195.

Reçu le 31.07.2004, accepté le 21.02.2006

\section{Resumen}

Gargouri A. Producción y composición de la leche de oveja: efectos de los aportes en lípidos protegidos

Se creó una base de datos sobre los efectos del aporte de lípidos protegidos (principalmente jabones de calcio a partir de aceite de palma), con el fin de cuantificar la respuesta de la producción y de la composición de la leche en las ovejas lecheras. De manera general, la incorporación de este tipo de suplemento no afectó la cantidad de la leche a comercializar, llevó a un aumento de las tasas butíricas $(+21 \%$ ) y a una depresión de las tasas proteicas $(-4 \%)$. Estas respuestas son muy variables y dependen de la cantidad de suplemento lipídico y del estadio de lactación de las ovejas. Las mejores respuestas se observaron con un nivel de ingestión de jabón cálcico cercano a 150 g/día/ oveja administrados al inicio de la lactancia. La composición en ácidos grasos de la leche fue casi siempre modificada por los lípidos protegidos, principalmente con una disminución de la proporción de los ácidos grasos cortos y medios y un crecimiento de los ácidos grasos largos. La velocidad de crecimiento de los corderos criados por ovejas bajo suplemento no fue modificada durante el periodo de lactación.

Palabras clave: Ovino - Leche de oveja - Lípido - Composición química - Cordero - Crecimiento - Túnez. 